\title{
Luxofractura cervical en niños: revisión bibliográfica y caso clínico
}

\author{
Juan Manuel Velasco Canziani, Santiago Saprizza, Nicolás Galli, Natalia Otero, Fernando García \\ CE.DEF.CO. Centro de Deformidades de Columna, Montevideo, Uruguay
}

\begin{abstract}
RESUMEN
Las luxofracturas son poco frecuentes, pero revisten mucha importancia a la hora de considerar el diagnóstico, el tratamiento y las posibles secuelas. Presentamos una revisión bibliográfica sobre luxofracturas cervicales en niños y el caso de una niña de 10 años que sufrió una luxofractura de C3-C4. La paciente fue sometida a una reducción abierta y una artrodesis de C3-C4, y controlada durante 2 años.
\end{abstract}

Palabras clave: Luxofractura cervical; niños; artrodesis; reducción cervical.

Nivel de Evidencia: IV

Cervical spine fracture-dislocations in children: literature review and clinical case report

\section{ABSTRACT}

Fracture-dislocations are a rare presentation, but their knowledge is critical in terms of diagnosis, treatment, and potential sequelae. We report a literature review on cervical spine fracture-dislocations in children and a case of a 10-year-old girl who sustained a C3-C4 fracture-dislocation. The patient underwent open reduction and arthrodesis at C3-C4, and a 2-year follow-up.

Key words: Cervical fracture-dislocations; children; arthrodesis; cervical reduction.

Level of Evidence: IV

\section{INTRODUCCIÓN}

Las lesiones traumáticas graves de la columna cervical son cuadros infrecuentes en los niños, y engloban a fracturas, luxaciones y luxofracturas de la columna vertebral. En los niños, las causas de estas lesiones pueden ser similares a las de los adultos, como accidentes en la vía pública o deportivos, pero la localización, la anatomía patológica, su potencial evolutivo y su tratamiento suelen diferir.

Estas lesiones afectan a un esqueleto en crecimiento con zonas cartilaginosas vertebrales aún no osificadas, es decir, zonas de debilidad en los niños y, en ocasiones, son difíciles de evaluar en las radiografías. Asimismo, la elasticidad ligamentaria es mayor, lo que permite más desplazamiento vertebral ante los traumatismos y puede provocar un cuadro neurológico. Se destaca la presencia de una entidad clínica llamada lesión de SCIWORA (Spinal Cord Injury Without Radiographic Abnormality) que corresponde a una lesión neurológica sin alteraciones óseas y es una entidad nosológica característica de los esqueletos en crecimiento. Estas particularidades desaparecen a medida que el esqueleto madura, hasta la adolescencia, cuando las lesiones son comparables a las del adulto.

Es preciso conocer la semiología radiológica de estas lesiones, sobre todo, la de la columna cervical superior, y saber que dichas lesiones pueden, en muchas oportunidades, plantear dudas y, en estos casos, se utilizan los diferentes criterios radiológicos para valorar la anatomía vertebral cervical descritos por Swischuk y cols., con el fin de complementar la información que ofrecen las radiografías, los estudios por imágenes, como las radiografías dinámicas para confirmar una inestabilidad ligamentaria, la resonancia magnética (RM) para valorar las lesiones de partes blandas (aparato ligamentario, disco intervertebral y contenido del canal medular) y la tomografía computarizada (TC) para evaluar el cuerpo vertebral y los procesos articulares. Estas exploraciones son las más eficaces para establecer un estudio preciso de las lesiones óseas, cartilaginosas y del contenido raquídeo. 
El tratamiento quirúrgico tiene indicaciones específicas, como la presencia de una lesión ligamentaria, las luxaciones facetarias, la lesión de SCIWORA o una alteración en la relación intervalo atlas-odontoides/intervalo arco posterior-odontoides, que determinarían una lesión en el complejo C1-C2. Estas entidades representan lo que se reconocería como lesiones graves de la columna cervical.

Es de vital importancia reconocer dichas lesiones para reducir, al mínimo, los trastornos del crecimiento secundarios a la cirugía (laminectomía, desperiostización, lesión fisaria, etc.). El seguimiento prolongado de estas lesiones es indispensable, debido al riesgo de trastornos del crecimiento a largo plazo.

En la mayoría de los casos, se observan deformaciones postraumáticas cuando la gravedad de la lesión inicial ha pasado inadvertida o el tratamiento ortopédico o la cirugía han sido inadecuados. Por lo tanto, prevenir estas deformaciones requiere conocer los elementos anatómicos responsables de la estabilidad y el crecimiento raquídeos.

\section{METODOLOGÍA}

Se realizó una búsqueda bibliográfica de artículos sobre luxofractura cervical en pediatría, publicados entre 2008 y 2018, en PubMed y Google Scholar, utilizando las siguientes palabras clave: "luxofractures", "children cervical fractures", "arthrodesis", "cervical reduction". Se encontraron y revisaron 15 estudios. Se halló que no hay consenso en cuanto a la elección del tratamiento según la edad y el tipo de lesión, en la población pediátrica. Durante muchos años, la disponibilidad de materiales y el conocimiento biomecánico limitados determinaron que muchas lesiones fueran tratadas de forma conservadora o usando técnicas de artrodesis in situ o fijación con cerclaje sublaminar, lo que requiere el uso de técnicas complementarias, como la fijación externa. Sin embargo, este tipo de tratamiento provoca consecuencias, como la alta tasa de seudoartrosis, la pérdida de reducción con las deformidades subsecuentes, así como una morbilidad adicional atribuida al halo cervical y a las cirugías de revisión. En los estudios, se reconoce que cada vez se utiliza más el tratamiento quirúrgico con reducción abierta y tornillos para las lesiones inestables y las que presentan compromiso del cordón medular, y que, además, pese a que no se dispone de instrumental específicamente diseñado para pediatría, con el tiempo, se ha observado que es una opción segura siempre que se tengan en cuenta las variaciones en la anatomía con respecto a los adultos y se realice una exhaustiva planificación preoperatoria para lo cual son muy útiles los estudios por imágenes (radiografías, TC y RM). No encontramos información sobre la necesidad o el beneficio de retirar el instrumental luego de la consolidación de la fractura o de la artrodesis.

Se presenta un caso clínico de una niña de 10 años que sufrió una luxofractura de C3-C4, y fue sometida a una reducción abierta y una artrodesis de C3-C4.

Los padres de la paciente autorizaron la cirugía, la toma de fotografías, el tratamiento y la publicación del caso mediante el consentimiento médico firmado en forma totalmente libre y con toda la información esclarecida.

Este estudio fue aprobado por el Comité de ética de nuestra institución.

\section{CASO CLÍNICO}

Niña de 10 años, procedente de un medio rural, producto de un embarazo a término, eugenésica, con buen desarrollo y crecimiento.

En julio de 2017, ingresa con un cuadro de cervicalgia secundario a un traumatismo directo contuso-cortante de cráneo en la zona occipital provocado por una hamaca metálica. La paciente está lúcida, mueve los cuatro miembros y los reflejos osteotendinosos son normales. Se detectan parestesias en C4-C5, en el miembro superior izquierdo. No presenta paresia ni déficits sensitivos en los cuatro miembros. Tiene dolor a la movilización del cuello, una herida contuso-cortante en el cuero cabelludo, en la región de la nuca, zona del occipital.

Llega al Servicio en tabla de traslado, con collar cervical de Filadelfia y vía venosa periférica con analgésicos. No había recibido corticoides intravenosos.

La radiografía simple, la TC y la RM muestran una luxofractura de $\mathrm{C} 3-\mathrm{C} 4$, con fractura de la articular inferior de C3 a izquierda y un desplazamiento anterior casi del 90\%. En la RM, además de valorar el sector ligamentario posterior, se visualiza el disco intervertebral sano y contenido por el ligamento longitudinal posterior sin desplazamiento hacia dentro del canal medular (Figuras 1-5).

La niña es operada de urgencia por un equipo de traumatólogos especialistas en cirugía vertebral.

Se colocó a la paciente en decúbito ventral, en mesa ortopédica radiolúcida, y con monitoreo electrofisiológico de la médula con potenciales motores (antes de comenzar la cirugía, durante todo el procedimiento y después, durante los cambios de posición de decúbito dorsal a ventral, y viceversa). Cabe aclarar que no se usó tracción mediante estribo de Mayfield. Para reducir, al mínimo, los riesgos durante la intubación orotraqueal, un endoscopista realizó la intubación. 


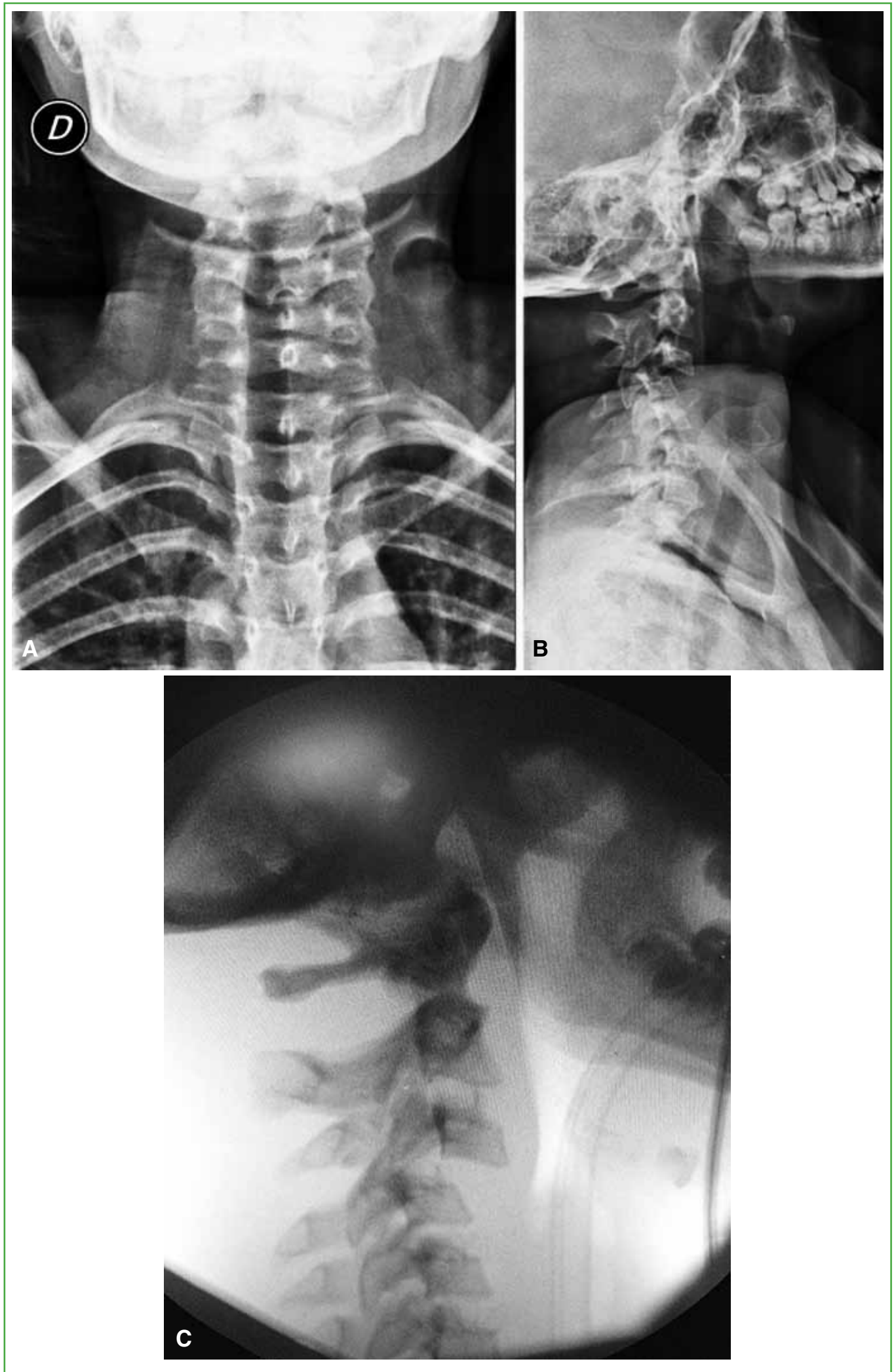

Figura 1. Radiografías iniciales, julio 2017. A. Imagen anteroposterior. B. Imagen lateral: se visualiza una listesis anterior de C3 de aproximadamente el 90\%. C. Radiografía lateral de columna cervical intraoperatoria. 


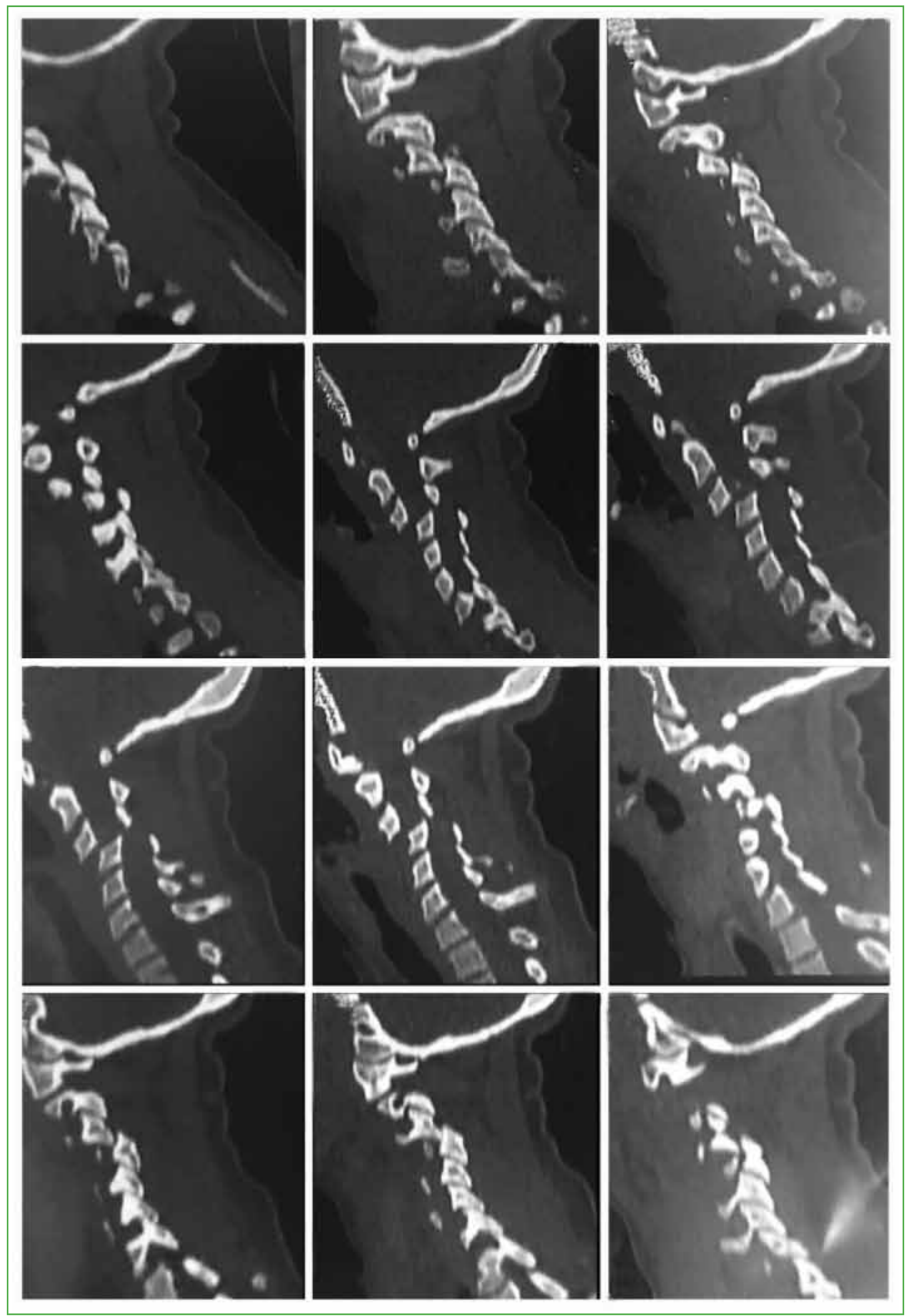

Figura 2. Tomografía computarizada tomada a la hora del traumatismo. Se observan una luxofractura facetaria bilateral y una listesis cercana al $100 \%$. 


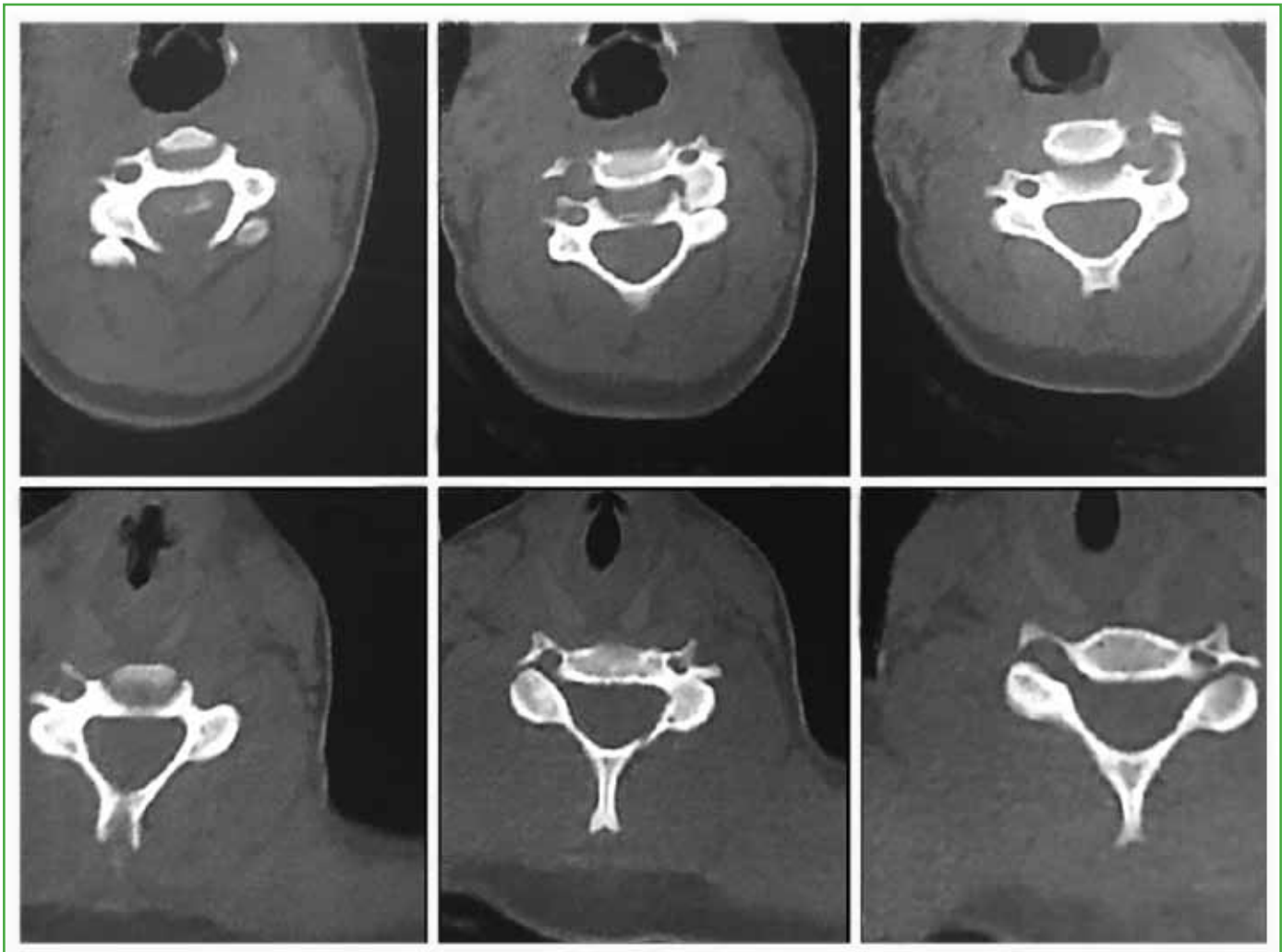

Figura 3. Tomografía computarizada, corte transversal. Se observa una luxofractura facetaria bilateral.
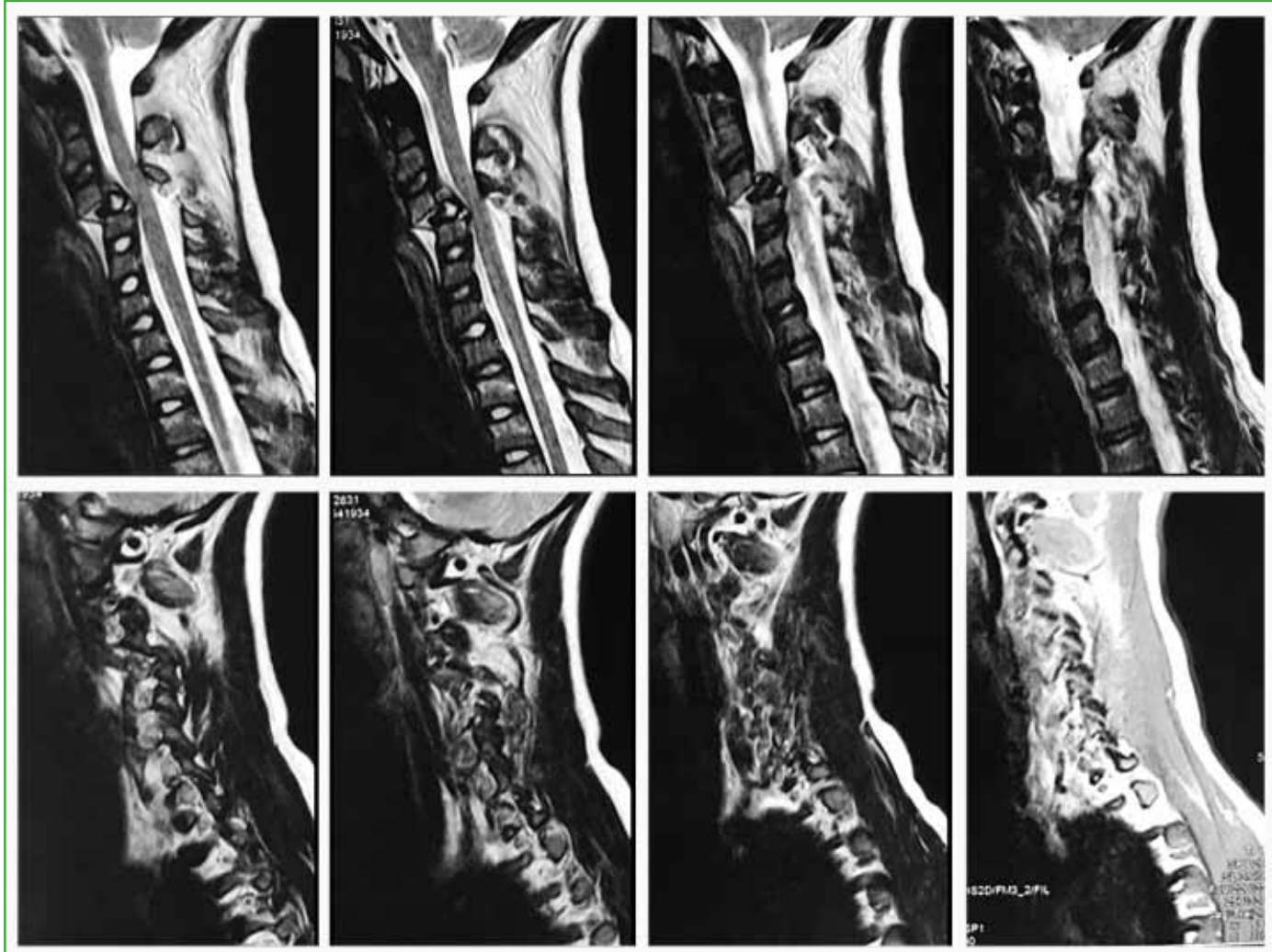

Figura 4. Resonancia magnética en secuencias T2. Se observan la luxación cervical y la hiperintensidad de la señal en el complejo ligamentario posterior. Ligamento longitudinal posterior sano, disco intervertebral contenido. 

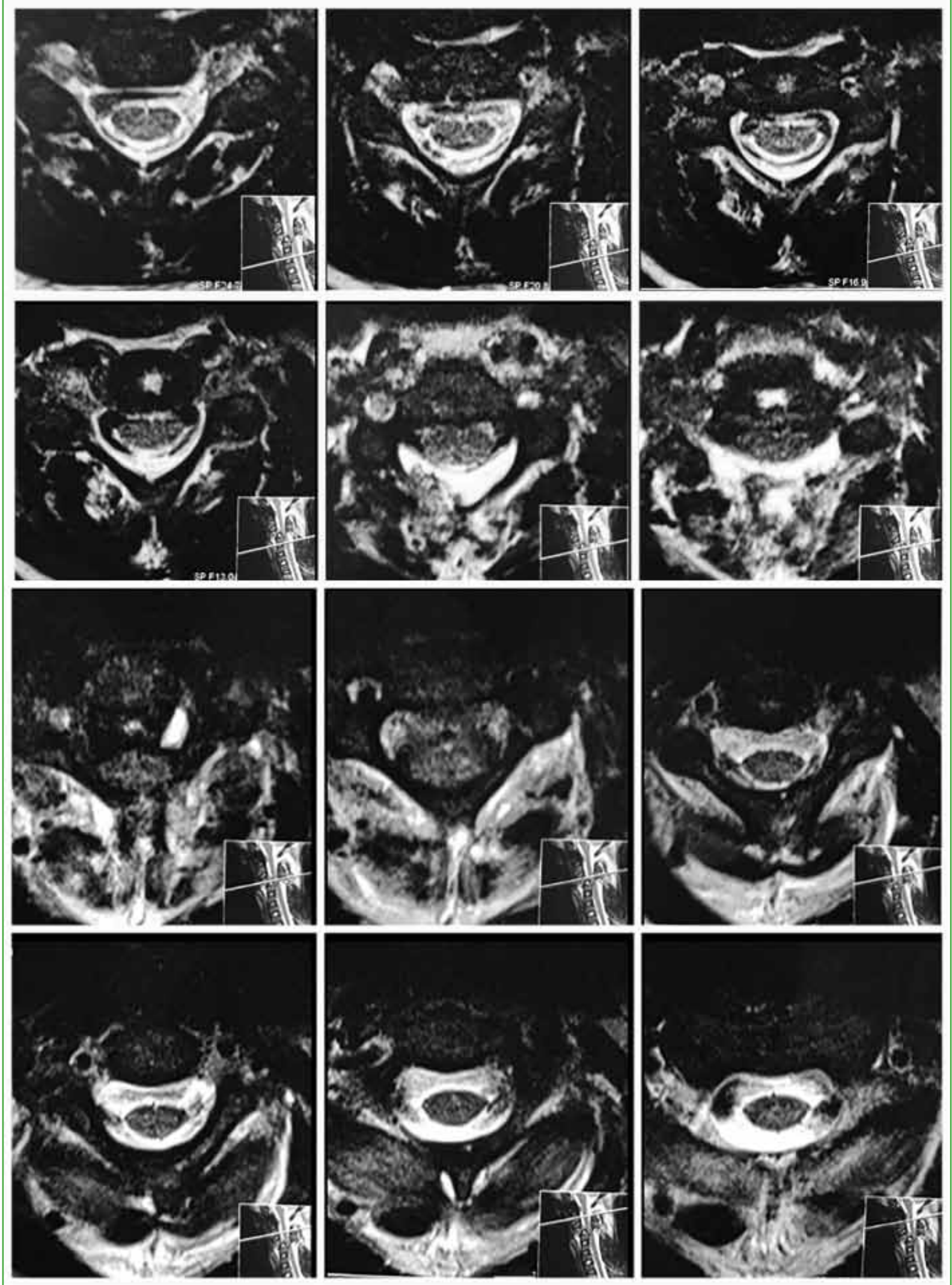

Figura 5. Resonancia magnética en secuencias T2. Se observa hiperintensidad de la señal en el complejo ligamentario posterior y en la apófisis espinosa de C3. 
Se efectuó un abordaje por longitudinal posterior centrado en C3. Se liberaron los músculos que exponen las láminas, hasta las articulares. Se visualizaron los ligamentos amarillo, supraespinoso e interespinoso rotos. Se detectó una fractura de la articular inferior de C3 a izquierda, en tanto que, del lado derecho, solo estaban luxadas las articulares. Se llevó a cabo la reducción mediante suave tracción y antes se resecó muy poco la articular superior de C4. Se tomó una radiografía intraoperatoria para valorar la correcta reducción y se procedió a colocar tornillos transarticulares C3-C4 bilateralmente y dos barras de titanio.

A continuación, se procedió a un nuevo control radiológico y a la colocación de un injerto autólogo de cresta ilíaca posterior. Cierre por planos y drenaje aspirativo por 24 horas.

Se le tomó una RM de columna cervical en el posoperatorio inmediato y luego ingresó en la Unidad de Cuidados Intensivos Pediátricos para monitorizacion y control. La RM se realizó fundamentalmente para valorar el canal cervical y la invasión del disco intervertebral intracanal. Este estudio mostró una excelente reducción sin compromiso ni invasión del canal medular, un disco intervertebral en su sitio, y los ligamentos intervertebral anterior y posterior sanos. Por este motivo, optamos por no realizar la vía anterior para la estabilización (Figura 6).

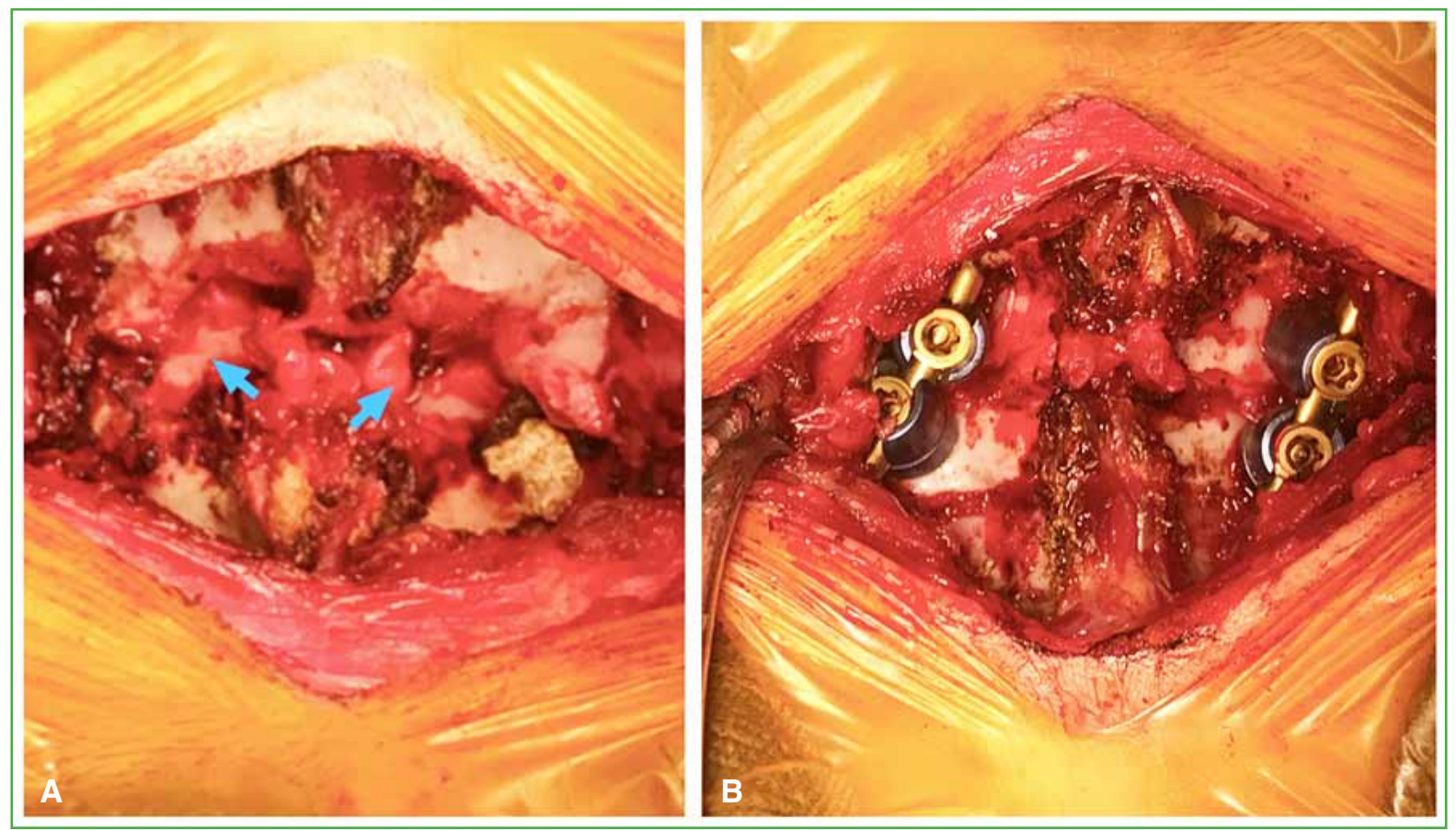

Figura 6. A. Luxación vertebral, articulares desnudas. B. Luxofractura reducida, tornillos articulares. 
Tras la cirugía, la paciente usó un collar de protección durante cuatro semanas. Los controles clínicos y radiológicos muestran una buena evolución. La paciente reanuda sus tareas habituales a los tres meses de la lesión (Figuras 7-10).
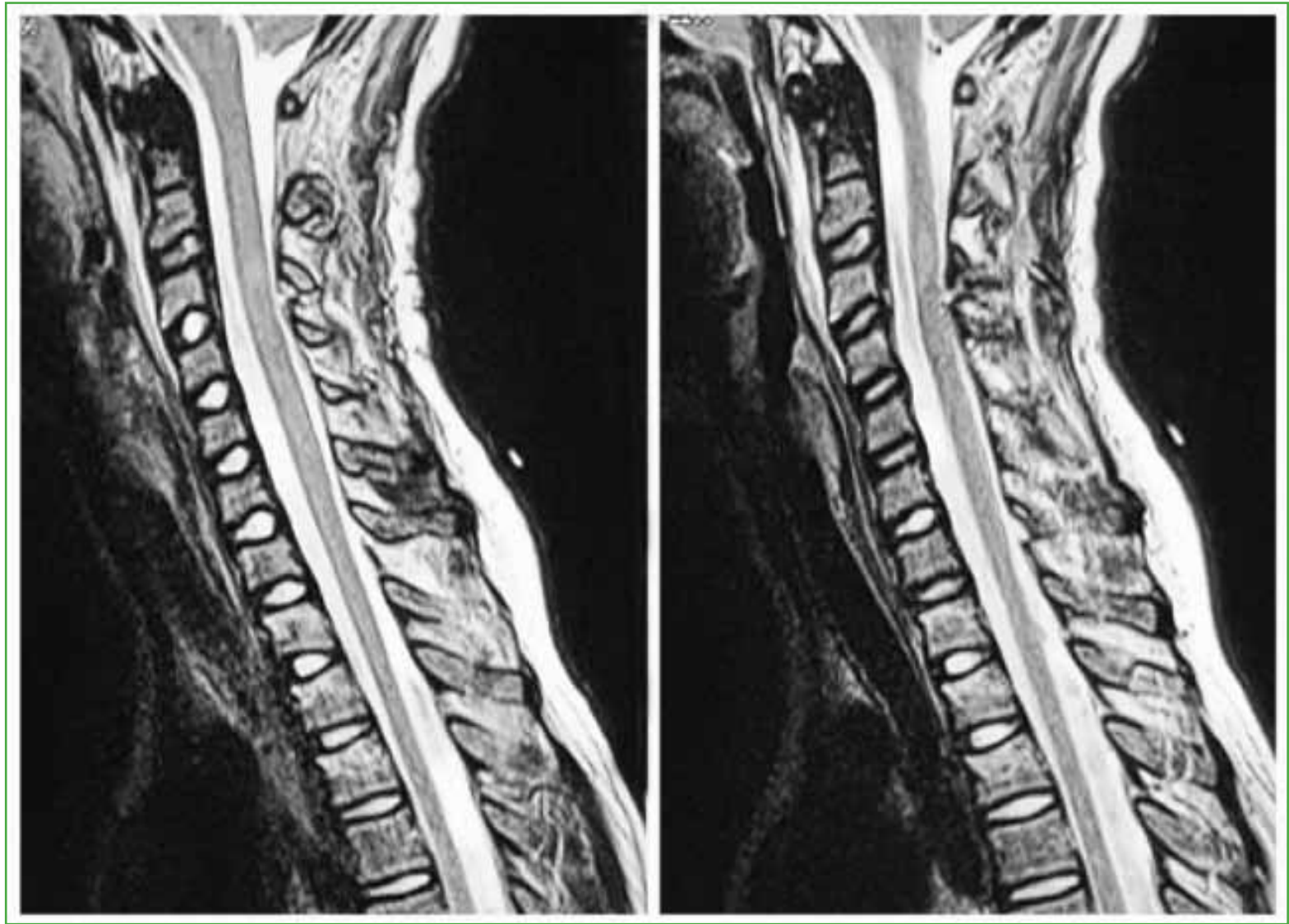

Figura 7. Resonancia magnética en el posoperatorio inmediato. Muy buena reducción. Disco intervertebral contenido por el ligamento longitudinal posterior, canal cervical amplio. 


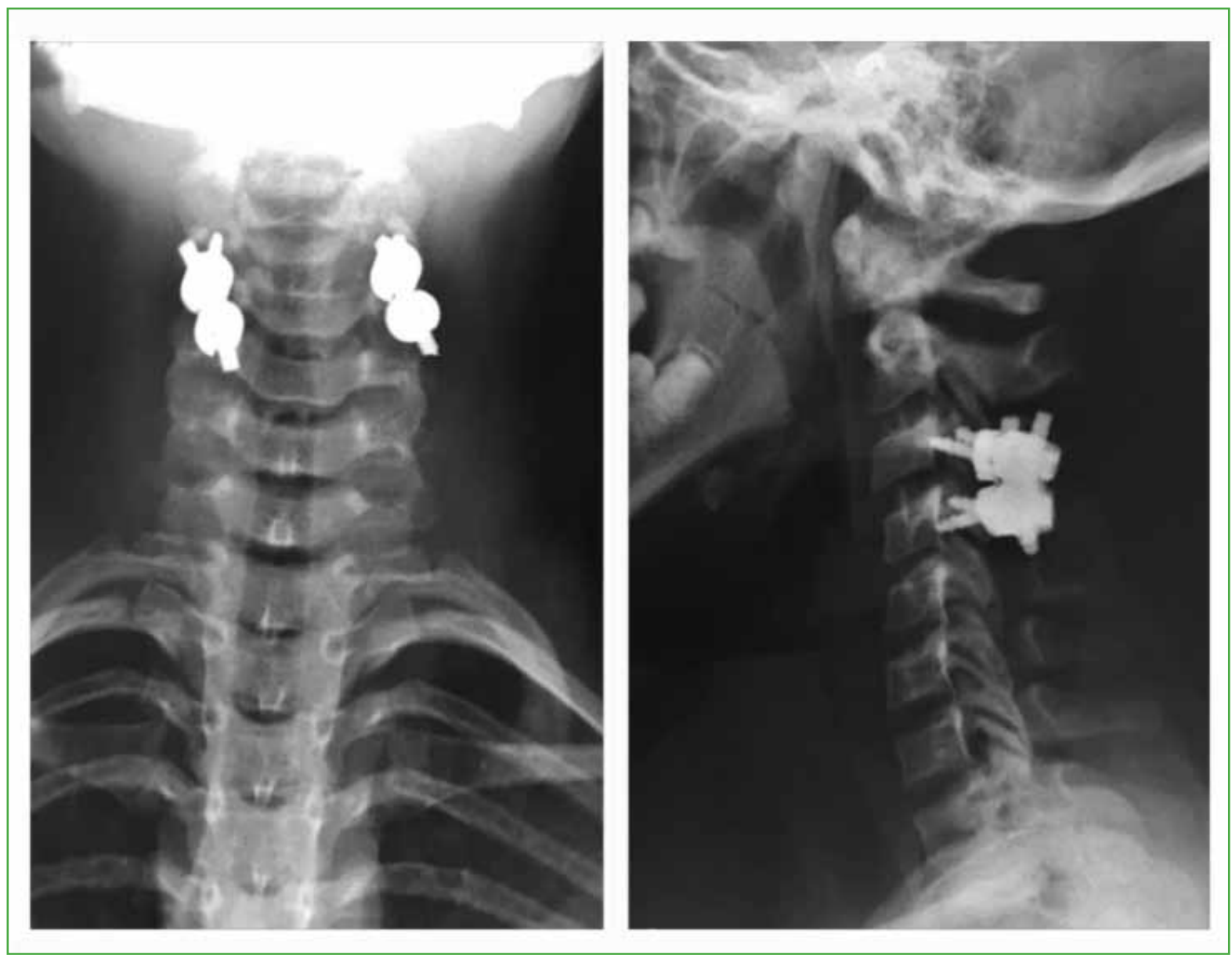

Figura 8. Radiografías tomadas al mes. 


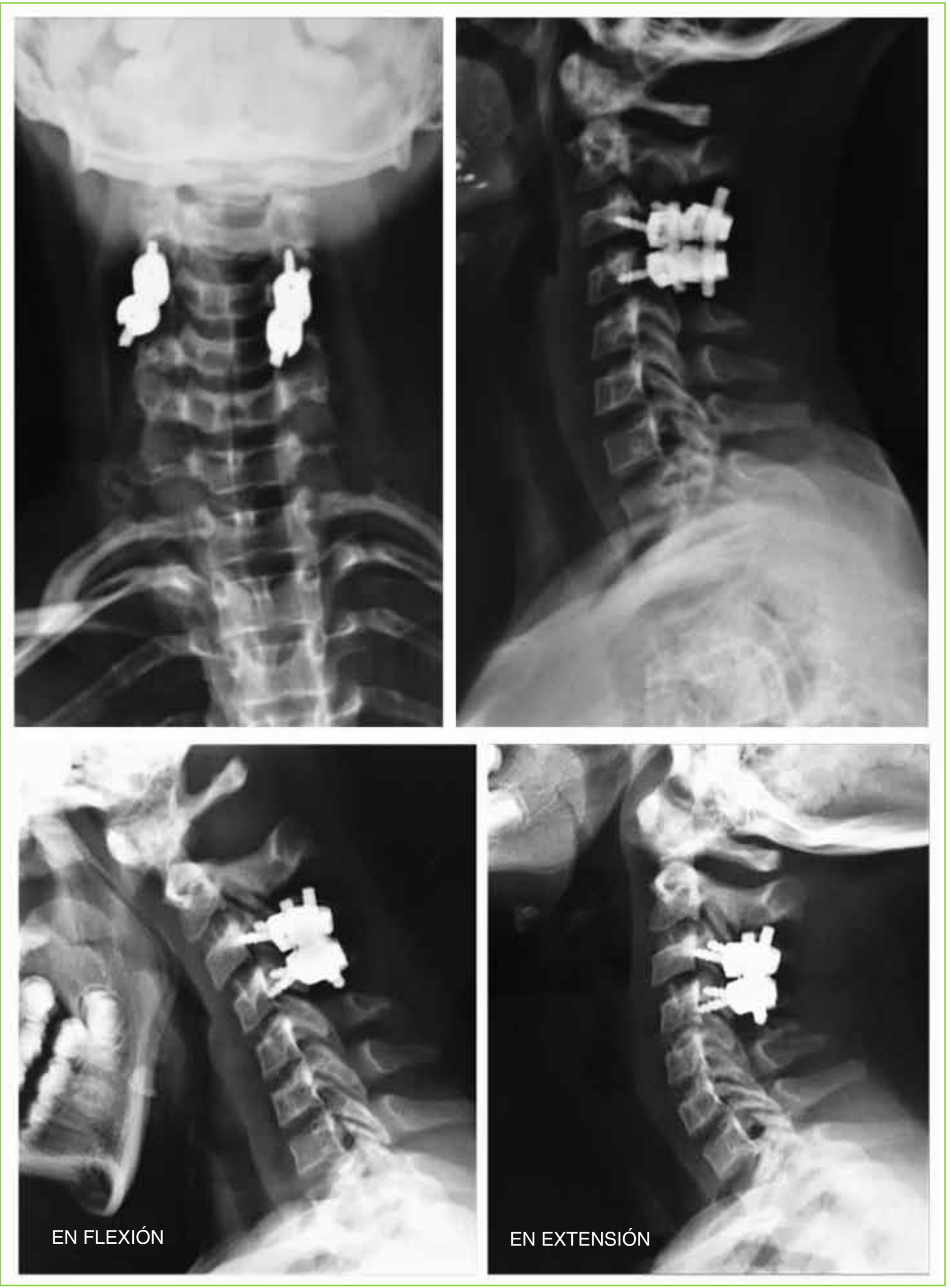

Figura 9. Radiografías funcionales de control a los tres meses. No se observan elementos de inestabilidad. 


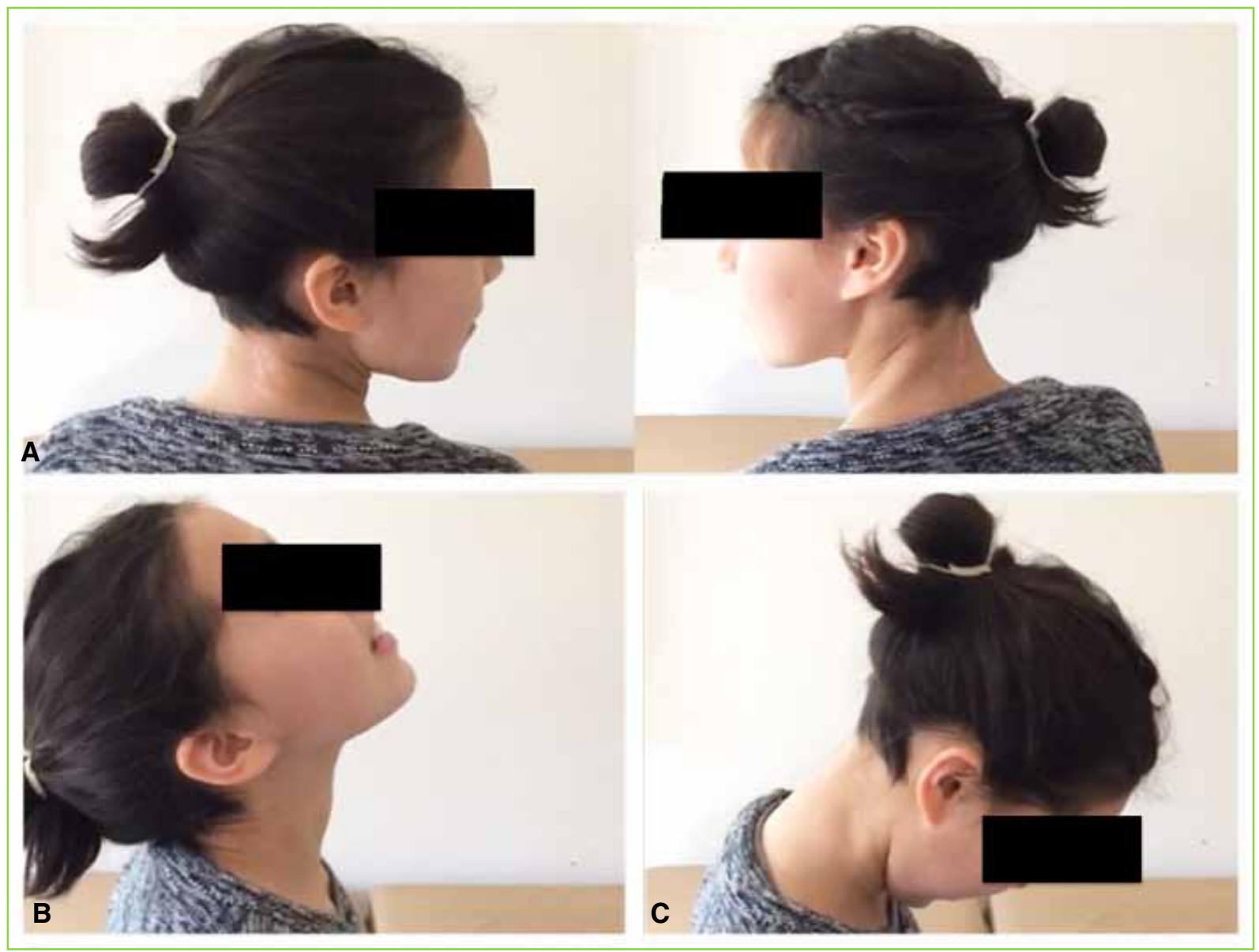

Figura 10. Imágenes tomadas a los tres meses de evolución. A. Rotaciones sin limitación. B. Extensión completa. C. Flexión completa sin dolor.

\section{DISCUSIÓN}

Los traumatismos afectan, con frecuencia, la región cervical en niños $<14$ años y la gran mayoría de las lesiones son inestables. Cuando ocurren en pacientes $<8$ años predominan en la región cervical alta, ${ }^{1}$ a causa de las características especiales anatómicas y fisiológicas de la zona y porque la cabeza pesa más en relación con el resto del cuerpo; además, se asocian, con menos frecuencia, a defectos neurológicos. A partir de los 10 años de edad, su comportamiento es igual que en los adultos. ${ }^{2-7}$

En lactantes y niños pequeños, predominan las lesiones del atlas, el axis y la columna cervical alta, debido al tamaño de la cabeza en relación con el cuerpo, la laxitud de los ligamentos y la diferencia de angulación de las articulares; además los músculos solo son un soporte en la pubertad. Las facetas de las tres primeras cervicales tienen una orientación horizontal que nunca será oblicua como en el resto de la columna cervical. En los niños pequeños, los procesos uncinados tienen un desarrollo incompleto y son planos y, por lo tanto, ineficientes para la rotación-flexión. Por otra parte, el punto de apoyo de los movimientos cervicales es más alto en los más pequeños que en adolescentes y adultos. ${ }^{7-11}$

Como la columna de los niños $<2$ años es inmadura y se osifica progresivamente, las lesiones tienden a ser separaciones epifisarias, luxaciones y fracturas de las placas de crecimiento más que verdaderas fracturas. Este mecanismo es válido para toda la columna, pero más para las fracturas de la odontoides. ${ }^{12-17}$ 


\section{CONCLUSIONES}

Presentamos a una niña de 10 años que sufrió una luxofractura de C3 con un desplazamiento cercano al 90\% y ASIA E al ingresar. En las primeras $24 \mathrm{~h}$, fue sometida a un abordaje por vía posterior con reducción abierta y artrodesis de $\mathrm{C} 3-\mathrm{C} 4$ mediante tornillos transarticulares. No tuvo complicaciones neurológicas posoperatorias, y su evolución fue muy buena sin limitaciones en la movilidad ni dolor. Creemos que esta paciente se vio beneficiada de un diagnóstico y un traslado rápidos, y un tratamiento quirúrgico en las primeras 24 h del traumatismo, que logró una correcta reducción y la estabilización de la lesión. Según el nuevo puntaje postraumático de columna cervical (dolor, movilidad, estado neurológico), el resultado fue excelente.

Nos queda por responder dos interrogantes: si una paciente de 10 años con esta lesión requiere una artrodesis y, si es necesario, retirar el instrumental durante la evolución.

Conflicto de intereses: Los autores no declaran conflictos de intereses.

\section{BIBLIOGRAFÍA}

1. Bollini G, Jove JL, Cottalorda J. Spinal trauma in children. En: Holtzman RNN, McCormick PC, Farcy J-PC. Spinal instability. New York, NY: Springer Verlag New York Inc.; 1993: 237-54.

2. Caffey J. The whiplash shaken infant syndrome: manual shaking by the extremities with whiplash-induced intracranial and intraocular bleedings, linked with residual permanent brain damage and mental retardation. Pediatrics 1974;54(4):396-403. PMID: 4416579

3. Dickman CA, Douglas RA, Sonntag VKH. Occipito-cervical fusion: posterior stabilization of the craniovertebral junction and upper cervical spine. BNI Q 1990;6(2):2-14.

4. Grabb PA, Pang D. Magnetic resonance imaging in the evaluation of spinal cord injury without radiographic abnormality in children. Neurosurgery 1994;35(3):406-14. https://doi.org/10.1227/00006123-199409000-00007

5. Nitecki S, Moir CR. Predictive factors of the outcome of traumatic cervical spine fracture in children. J Pediatr Surg 1994;29(11):1409-11. https://doi.org/10.1016/0022-3468(94)90131-7

6. Arlet V, Rigault P, Padovani JP, Finidori G, Touzet, P, Janklievicz P. Instability and misdiagnosed or neglected dislocations of the upper cervical spine in children. Rev Chir Orthop Reparatrice Appar Mot 1992;78(5):300-11. [En francés] PMID: 1289981

7. Bailey DK. The normal cervical spine in infants and children. Radiology 1952;59(5):712-9. https://doi.org/10.1148/59.5.712

8. Cattell HS, Filtzer DL. Pseudosubluxation and other normal variation in the cervical spine in children. A study of one hundred and sixty children. J Bone Joint Surg Am 1965;47(7):1295-309. PMID: 5837630

9. Netter RH. The CIBA collection of medical illustration, Vol. 8 Musculo-skeletal system, part 1. Anatomy, physiology and metabolics disorders. Summit, NJ: CIBA-Geygi; 1987.

10. Sherk HH, Schut L, Lane JM. Fractures and dislocations of the cervical spine in children. Orthop Clin North Am 1976;7(3):593-604. PMID: 958685

11. Price AE. Unique aspects of pediatric spine injuries. En: Errico TJ, Baver RD, Waugh T. Spinal trauma. Philadelphia: Lippincott Company; 1991:581-625.

12. Vigouroux RP, Baurand C, Choux M, Pellet W, Guillermain P. Les traumatismes dus rachis cervical chez l'enfant. Neurochirurgie 1968;14(6):689-702. [En francés] PMID: 5688169 
13. Von Torklus D, Gehle W. The upper cervical spine. Regional anatomy, pathology and traumatology. En: Verlag GT (ed.). A systemic radiological atlas and textbook. New York: Grune \& Stratton; 1972:2-91.

14. Nypaver M, Treolar D. Neutral cervical spine positioning in children. Ann Emerg Med 1994;23(2):208-11. https://doi.org/10.1016/s0196-0644(94)70032-X

15. Sontag VK, Dickman CA. Operative management of occipito cervical and atlantoaxial instability. En: Holtzman RNN, Mccormick PC, Farcy JP (eds.). Spinal instability. Springer Verlag; 1993:255-94.

16. Brockmeyer D, Apfelbaum R, Tippets R, Walker M, Carey L. Pediatric cervical spine instrumentation using screw fixation. Pediatr Neurosurg 1995;22(3):147-57. https://doi.org/10.1159/000120893

17. Bucholz RD, Cheung KC. Halo vest versus spinal fusion for cervical injuries: evidence for an outcome study. J Neurosurg 1989;70(6):884-92. https://doi.org/10.3171/jns.1989.70.6.0884 